\title{
Development of a Mecanum-Wheeled Mobile Robot for Dynamic- and Static-Obstacle Avoidance Based on Laser Range Sensor
}

\author{
Musa Matli ${ }^{1}$ D, Ahmet Albayrak ${ }^{2}$ D, and Raif Bayir ${ }^{3}$, \\ ${ }^{1}$ Graduate Education Institute, Department of Mechatronics Engineering, Karabuk University, Karabuk, \\ Turkey \\ ${ }^{2}$ Faculty of Technology, Department of Computer Engineering, Duzce University, Duzce, Turkey \\ ${ }^{3}$ Faculty of Technology, Department of Mechatronics Engineering, Karabuk University, Karabuk, Turkey
}

\section{]jfis}

\begin{abstract}
This study aims to present an idea about the practical consequences of using mobile robots with Mecanum wheels. For mobile robots, an approach is proposed to avoid obstacles without location and map information. This approach is presented using a series of developed solutions. This article shares the process on how a set of discussed conceptual methodologies can be applied as well as their practical results. This method is provided using fuzzy logic and gap tracking. LIDAR is used to recognize obstacles around the mobile robot. By using the LIDAR, the robot detects gaps around it and moves according to fuzzy logic. The fuzzy logic consists of three inputs, an output, and 45 rules. The first of the membership functions represents the membership function that replaces the obstacle. The second membership function calculates the distance to the obstacle. The final login membership function is used to determine the angle between the obstacle and robot view. The output membership function represents the membership function that moves the robot. The results are analyzed under three different scenarios with five different experiments for each scenario. The results show that the mobile robot can avoid obstacles without location and map information. We believe that the proposed method can be used in mobile robots such as guard and service robots.
\end{abstract}

Keywords: Fuzzy logic, Mobile robot, Follow-the-gap method, Mecanum wheel

Received: May 7, 2020

Revised : Jun. 8, 2020

Accepted: Sep. 14, 2020

Correspondence to: Raif Bayir

(rbayir@karabuk.edu.tr)

@The Korean Institute of Intelligent Systems

(c) This is an Open Access article distributed under the terms of the Creative Commons Attribution Non-Commercial License (http://creativecommons.org/licenses/ by-nc/3.0// which permits unrestricted noncommercial use, distribution, and reproduction in any medium, provided the original work is properly cited.

\section{Introduction}

Mobile robots move within predefined areas, instead of along specially defined areas. They are capable of autonomous movement without a human operator [1,2]. When they move within a working area, they must learn the environment and carry out their movement according to the obstacles. Their mobility varies depending on the wheel structure. In the 1970s, Bengt Ilon developed the Mecanum wheel. Mecanum wheels greatly increase the mobility of mobile robots [3]. On the other hand, the circumference-detection method and increase in mobility make robot control difficult. Because of the variable dynamics of the environment and the mobile structure of the robot, intelligent control methods are needed. The control method determines how the robot will perform a motion plan by interpreting the data from the sensors [4]. Detecting the environment/obstacles and determining the direction of movement relative to these obstacles are the main problems in mobile-robot control. Many studies have been 


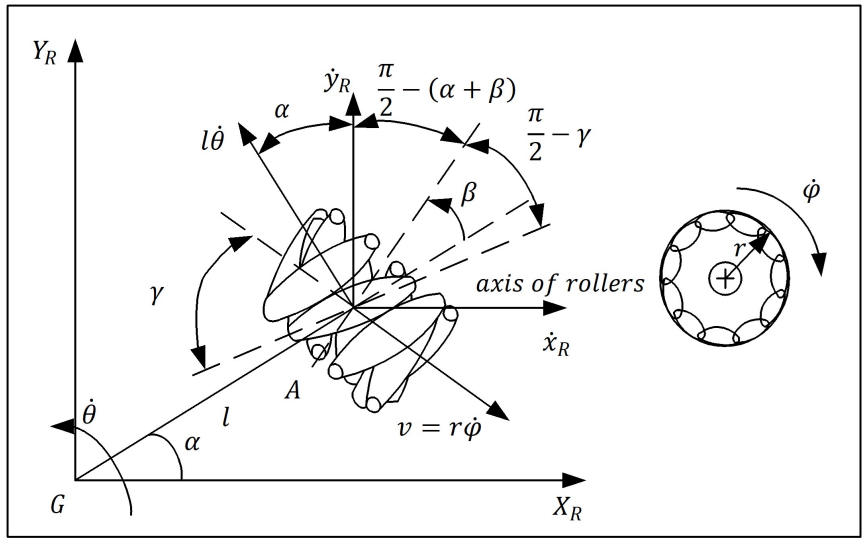

Figure 1. Parameters of the Mecanum wheel.

presented in the literature in this field, and many methods have been proposed. In addition to the image-based methods for obstacle detection, laser-based distance sensors and sonar sensors are used. The main obstacle-detection methods are enumerated as follows: artificial potential field method $[4,5]$, vector field histogram [6, 7], bug algorithm [8], follow-the-gap method [9], fuzzy logic [10], and neural network [11].

Mecanum wheel is a traditional wheel with a series of rollers fastened around it. Each of these rollers has an axis of rotation of $45^{\circ}$ with the plane of the wheel and $45^{\circ}$ with a line that passes through the center of the cylinder parallel to the axis of rotation of the wheel. Knowledge of the Mecanum wheel structure, mobility, and mathematical equations is important to develop a mobile-robot platform with Mecanum wheels [12, 13].

The Mecanum wheel consists of a Swedish wheel and a fixed standard wheel with passive rollers attached to the circumference of the wheel (Figure 1). Mecanum wheel is a type of Swedish wheel with $\gamma=45^{\circ}$, where $\gamma$ is the angle between the wheel plane and rotation axis of the passive rollers [14].

In the present study, a mobile-robot platform with four Mecanum wheels was developed. A laser-based distance-detection sensor was installed on the mobile robot. To prevent moving as well as avoid mobile obstacles in a closed environment, the follow-the-gap and fuzzy-logic methods were used together. The experiments demonstrated that successful results could be obtained using these methods. Fuzzy controllers have become very popular in mobile-robot applications because of the use of linguistic variables. They are used to process and classify complex data. Mobile-robot motion control is a complex system. Fuzzy logic is suitable for mobile-robot movements. In the current study, the robot-motion behavior was mainly controlled [15]. Mobility is limited in four-wheeled mobilerobot platforms without Mecanum wheels. Differential-type mobile robots must perform turn motions while avoiding obstacles. Compared with mobile robots with Mecanum wheels, the differential-type mobile robots lose more time while avoiding obstacles. In our study, the robot scanned its environment using a LIDAR sensor. In systems that operate using a stereo camera placed in one direction, the left and right areas of the robot cannot be controlled. Sensors are placed in these areas, and these sides are controlled. In our study, the robot environment was controlled using a LIDAR sensor [16].

The contribution of this study to the literature is its use of fuzzy logic and follow-gap- methods together in the mobilerobot motion control. In this context, it offers an alternative for system operation based on navigation by providing the ability to avoid obstacles in indoor areas without the need for information such as the location of obstacles, map information, and robot location. Mobile-robot platforms with Mecanum wheels provide an omnidirectional advantage in avoiding obstacles. The proposed system can be used in mobile robots that operate in a narrow area. We believe them to be advantageous in warehouse and industrial transportation processes owing to their omnidirectional linear movement. The robot ability to move without disturbing the reference angle does not change the viewpoint of the equipment. Thus, it can also be used as an indoor guard or service robot. The proposed approach may be preferred because it is cheaper than the navigation-based systems.

This study is structured by explaining the kinematic model of the mobile robot with Mecanum wheel and discussing the design and production after the literature review is provided. Next, the method for detecting obstacles and gap widths is described. The design of the fuzzy-logic classifier and its relationship to the gap widths are provided. Experimental studies are explained, and the paper is completed with conclusions and evaluation. Design and production of Mecanum wheeled mobile robot.

\subsection{Kinematics of a Robot with Four Mecanum Wheels}

A Mecanum wheel mounted on a mobile robot with local coordinate frame $\{R\}: X_{R} Y_{R} Z_{R}$ is shown in Figure 1. Point $A$ is the center of the wheel, and the other geometrical parameters are defined as follows: $\alpha$ is the angle of the $G A$ vector relative to $X_{R}$ from origin $G$ to point $A$, and $\beta$ is the angle between the main wheel angle and $G A$ vector. The distance from geometric center $G$ to wheel center $A$ is $l$, and the main wheel radius is 
Table 1. Mecanum wheel parameters

\begin{tabular}{cccc}
\hline Wheels & $\boldsymbol{\alpha}_{\boldsymbol{i}}$ & $\boldsymbol{\beta}_{\boldsymbol{i}}$ & $\boldsymbol{\gamma}_{\boldsymbol{i}}$ \\
\hline 1 & $\tan ^{-1}(b / a)$ & $-\tan ^{-1}(b / a)$ & $(\pi / 2+\pi / 4)$ \\
\hline 2 & $\pi-\tan ^{-1}(b / a)$ & $\tan ^{-1}(b / a)$ & $-(\pi / 2+\pi / 4)$ \\
3 & $\pi+\tan ^{-1}(b / a)$ & $-\tan ^{-1}(b / a)$ & $(\pi / 2+\pi / 4)$ \\
4 & $2 \pi-\tan ^{-1}(b / a)$ & $\tan ^{-1}(b / a)$ & $-(\pi / 2+\pi / 4)$ \\
\hline
\end{tabular}

r. $\dot{\varphi}$ and $\dot{\varphi}_{S W}$ are the rotational speeds of the main wheel and passive rollers in contact with the ground, respectively [17].

By assuming that the contact point between the mechanical wheel and ground is the sudden center of rotation, the contact is in a simple rolling state without sliding. Then, the speed of wheel center $A$ along the tangential direction is $r \dot{\varphi}$, as shown in Figure 1. Thus, the speed component of wheel center $A$ along the axis of the contacting wheels is $r \dot{\varphi} \cos \gamma$. The instantaneous displacement rate of the robot under the $\{R\}$ conditions in the local frame is $\left[\begin{array}{ll}\dot{x}_{R} & \dot{y}_{R}\end{array}\right]^{T}$. The rotational speed around the $Z_{R}$ axis is $\theta$. The speed of point $A$, which is the center of the wheel, can be calculated by adding displacement velocity vectors $\dot{x}_{R}$ $\dot{y}_{R}$ and relative speed $l \dot{\theta}$ due to the rotation speed, as shown in Figure 1 [14].

We assume that the four Mecanum wheels are mounted at equal distances. Thus, Table 1 is taken into account, and the motion equation is expressed as Eq. (1).

$$
\begin{aligned}
{\left[\begin{array}{c}
\dot{\varphi}_{1} \\
\dot{\varphi}_{2} \\
\dot{\varphi}_{3} \\
\dot{\varphi}_{4}
\end{array}\right]=} & -(\sqrt{2} / r)\left[\begin{array}{ccc}
\sqrt{2} / 2 & \sqrt{2} / 2 & l \sin (\pi / 4-\alpha) \\
\sqrt{2} / 2 & -\sqrt{2} / 2 & l \sin (\pi / 4-\alpha) \\
-\sqrt{2} / 2 & -\sqrt{2} / 2 & l \sin (\pi / 4-\alpha) \\
-\sqrt{2} / 2 & \sqrt{2} / 2 & l \sin (\pi / 4-\alpha)
\end{array}\right] \\
\times & {\left[\begin{array}{ccc}
\cos \theta & \sin \theta & 0 \\
-\sin \theta & \cos \theta & 0 \\
0 & 0 & 1
\end{array}\right]\left[\begin{array}{c}
\dot{x}_{I} \\
\dot{y}_{I} \\
\dot{\theta}
\end{array}\right] . }
\end{aligned}
$$

In this equation, $\alpha=\tan ^{-1}(b / a)$ is considered. The horizontal distance of the robot from the center point to the center of the wheel is $a$, and the vertical distance is $b$. The Jacobian matrix is defined by Eq. (2).

$J=\left[\begin{array}{ccc}\sqrt{2} / 2 & \sqrt{2} / 2 & l \sin (\pi / 4-\alpha) \\ \sqrt{2} / 2 & -\sqrt{2} / 2 & l \sin (\pi / 4-\alpha) \\ -\sqrt{2} / 2 & -\sqrt{2} / 2 & l \sin (\pi / 4-\alpha) \\ -\sqrt{2} / 2 & \sqrt{2} / 2 & l \sin (\pi / 4-\alpha)\end{array}\right] \cdot\left[\begin{array}{ccc}\cos \theta & \sin \theta & 0 \\ -\sin \theta & \cos \theta & 0 \\ 0 & 0 & 1\end{array}\right]$.

According to the aforementioned equations, the advanced kine-

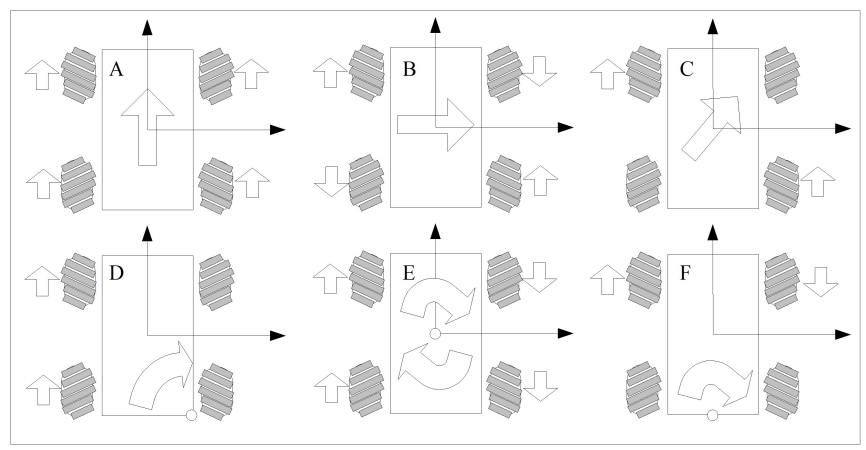

Figure 2. View of the mobile-robot mobility with a Mecanum wheel.

matic equation of the four-Mecanum-wheeled mobile robot is expressed as Eq. (3).

$$
\left[\begin{array}{c}
\dot{x}_{I} \\
\dot{y}_{I} \\
\dot{\theta}
\end{array}\right]=-(\sqrt{2} / 2) r J^{+}\left[\begin{array}{c}
\dot{\varphi}_{1} \\
\dot{\varphi}_{2} \\
\dot{\varphi}_{3} \\
\dot{\varphi}_{4}
\end{array}\right] .
$$

Here, $J^{+}=\left(J^{T} J\right)^{-1} J^{T}$ is the inverse of $J$. Figure 2 shows the movement capabilities of the Mecanum-wheeled mobile robot.

The kinematic and dynamic properties of the Mecanumwheeled mobile robots show that they can achieve omnidirectional movements. Some movements of the Mecanum-wheeled mobile robot are shown in Figure 2. In this study, A, B, C, E, and their inverse motion types are used, as shown in Figure 2. By using these movement types, static and dynamic obstacles are avoided.

\subsection{Design of a Mecanum-Wheeled Mobile Robot}

The mobile-robot platform consists of four direct current (DC) motors, LIDAR, motor drives, minicomputer, battery, Mecanum wheels, and body. The general representation of the system is shown in Figure 3. The top LIDAR and touch screen are positioned at the top of the robot.

The production-development process of the robot from the design stage is shown in Figure 4.

The DC motors are driven by RoboClaw $2 \times 15$ A motor drives. Each drive is also connected to a minicomputer. The Intel NUC5i7RYB minicomputer is used as the system controller. The minicomputer sends the necessary control signals to the motor drives. The LIDAR sensor is connected to the minicomputer. The minicomputer interprets the data from LIDAR and generates the necessary control signals. The employed driver, computer, motor, and sensors are shown in Figure 5. 


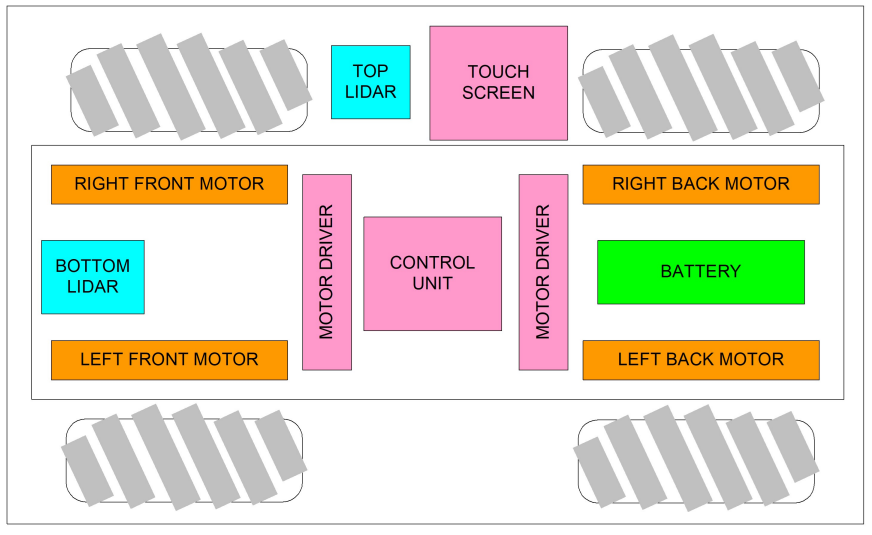

Figure 3. View of the system structure.

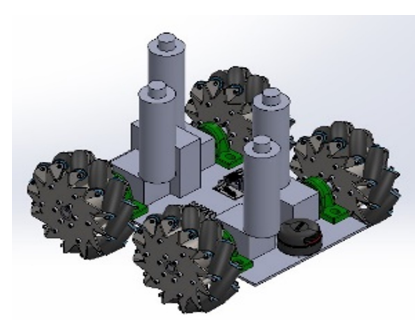

(a)

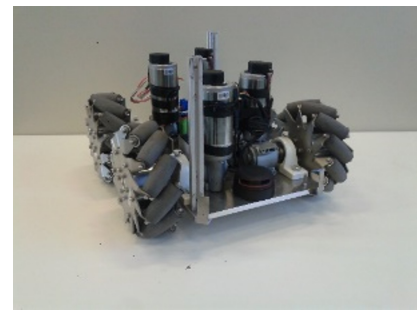

(c)

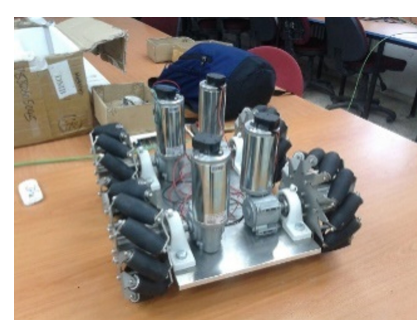

(b)

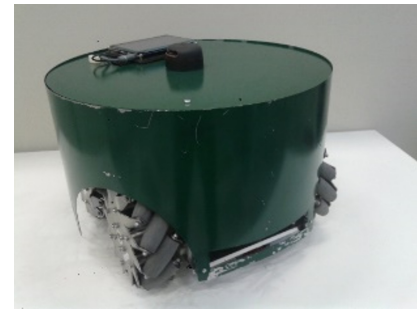

(d)
Figure 4. Images of the manufactured mobile robot from simulation to the final assembly: (a) robot design, (b) mechanical assembly, (c) electrical design, and (d) final assembly.

This study uses Mecanum wheels whose diameter, width, and carrying capacity are $203 \mathrm{~mm}, 78 \mathrm{~cm}$, and $150 \mathrm{~kg}$, respectively. The robot movement is provided by a $24-\mathrm{V} 95-\mathrm{W} 220-\mathrm{r} / \mathrm{min}$ DC gearmotor. A $2 \times 15$ A motor driver is used as the driver of the motors. RP A2 LIDAR is used to detect obstacles and the environment. It has a $360^{\circ}$ detection capacity. It provides 8000 point distance information per second. Its detection distance is $20 \mathrm{~m}$.

\section{Detecting Obstacles and Gaps}

Obstacles are detected using the data received from the LIDAR. The distance data are filtered by considering zero for obstacles

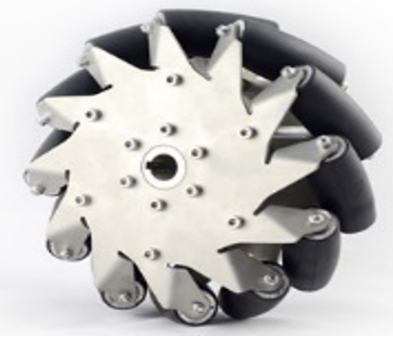

(a)

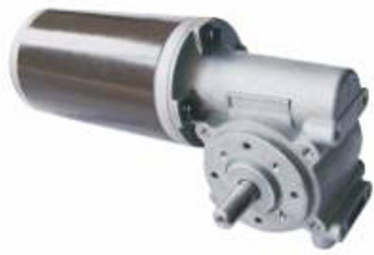

(c)

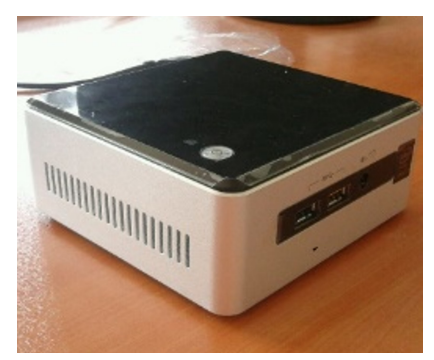

(b)

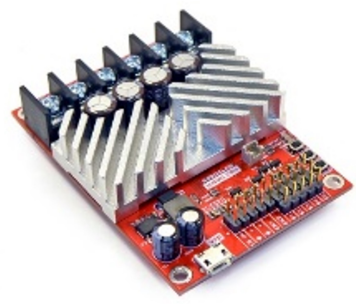

(d)

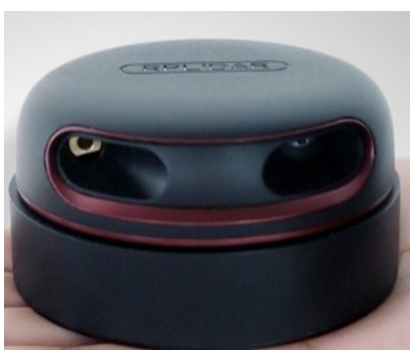

(e)

Figure 5. Equipment used in this study. (a) Mecanum wheel, (b) Intel NUC, (c) DC motor, (d) Motor drive, and (e) LIDAR.

that are farther than $1 \mathrm{~m}$ and considering the distance for obstacles that are less than $1 \mathrm{~m}$. In the filtered data, a zero value indicates gaps, whereas data closer than $1 \mathrm{~m}$ indicate obstacles. In this case, values from zero and lower provide the start and end points of the obstacles. The gap width is calculated by finding the start and end points of the obstacles. Three different situations occur between the robot and obstacles, which are shown in Figure 6.

Figure 6(a) shows that the robot is positioned between the obstacles. Here $\mathrm{d} 1$ and $\mathrm{d} 2$ indicate the distances between the boundaries of the obstacles and the robot measured by the LIDAR. The angles of the boundaries of the obstacles with the robot are expressed as a1 and a2. The distance of the obstacle boundaries to the axis that passes perpendicular to the robot is expressed as $x 1$ and $x 2$. We conclude that if the sum of $x 1$ and $\mathrm{x} 2$ is greater than the width of the robot, the robot can pass. 


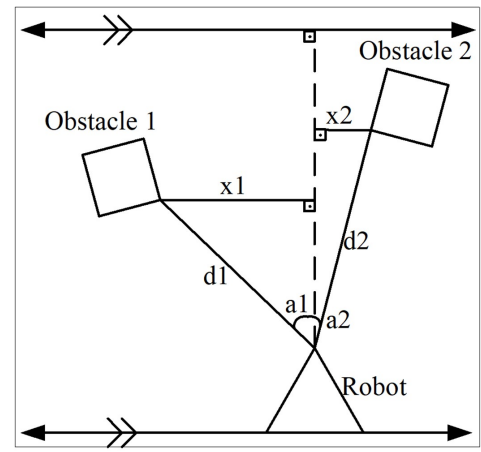

(a)

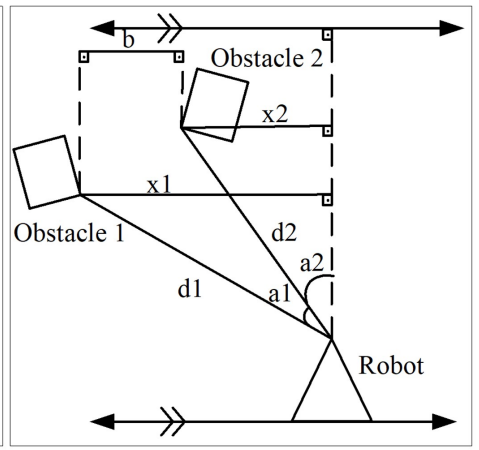

(b)

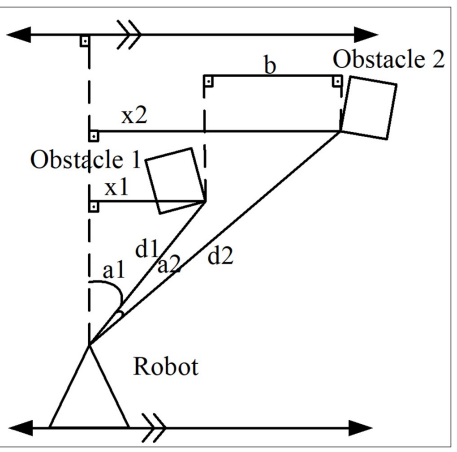

(c)

Figure 6. Situations between obstacles and the robot: (a) if robot middle of obstacle, (b) if robot right of obstacle, and (c) if robot left of obstacles.

Eq. (4) is used to calculate the gap width. Figure 6(b) shows the robot at the right side of the obstacles. In this case, the gap width to be calculated is given by Eq. (5). Figure 6(c) shows that the robot is located at the left side of the obstacles. In this case, Eq. (6) is used to calculate the gap width.

$$
\begin{aligned}
& x 1=d 1 / \sin (a 1), \\
& x 2=d 2 / \sin (a 2),
\end{aligned}
$$

Gap width $=x 1+x 2$,

$$
\begin{aligned}
& x 1=d 1 / \sin (a 1), \\
& x 2=d 2 / \sin (a 2),
\end{aligned}
$$$$
\text { Gap width }=x 1-x 2
$$$$
x 1=d 1 / \sin (a 1),
$$$$
x 2=d 2 / \sin (a 2),
$$

Gap width $=x 2-x 1$.

The three situations are evaluated according to the angle of the obstacle boundaries. More than one gap may exist because of the presence of more than one obstacle. In this case, all gap widths are calculated, and the maximum width is taken into account. If the width with the largest value is greater than the width of the robot, the robot is guided into that gap. The robot is guided to the midpoint of the gap during the orientation process. Calculation of the midpoint of the gap is performed using Eq. (7).

Gap center angle

$=\frac{\text { Gap right boundary angle }+ \text { Gap left boundary angle }}{2}$.

\section{Design of a Fuzzy Logic Classifier}

The robot starts to move by calculating the center angle of the gap. It must also avoid obstacles when moving toward the center of the gap. In this case, a fuzzy logic classifier is used to avoid obstacles and move the robot to the center of the gap. The fuzzy logic classifier is designed with three inputs and one output. The center angle of the gap, the closest obstacle angle, and the closest obstacle distance are given as input. The robot-movement type is determined by the output. Input values indicate where the robot is located in the gap, at which side of the obstacle, and how close it is to the center of the gap. Figure 7 shows the fuzzy logic classifier membership functions.

The position of the gap center relative to the robot is expressed by linguistic variables, namely, left, middle, and right. The viewing angle of the robot is $70^{\circ}$ from the center to the right and $-80^{\circ}$ to the left. The scanning angle of the robot is

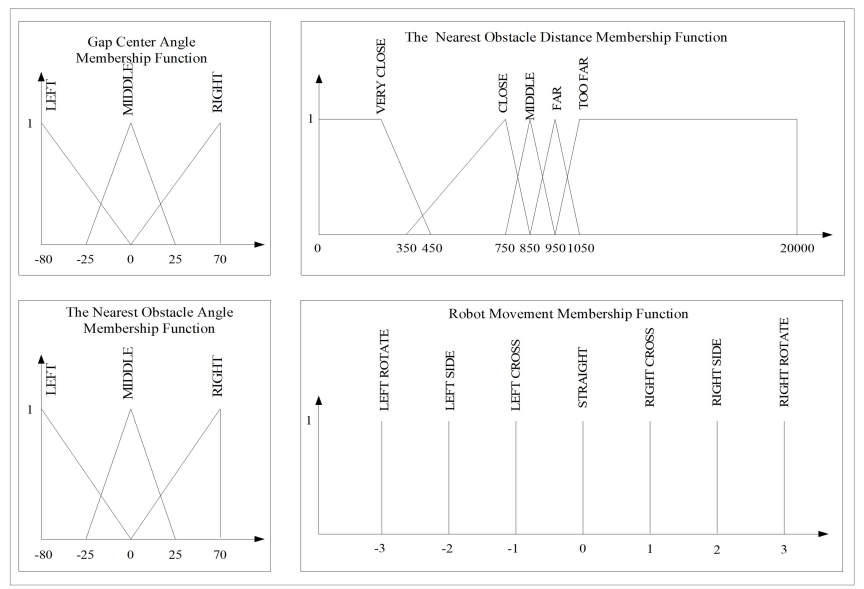

Figure 7. Input and output membership functions. 
the same as these angles. The angle of the nearest obstacle is expressed by linguistic variables, i.e., left, middle, and right. The third entry is the distance from the nearest obstacle to the robot. The distance of the nearest obstacle is evaluated under five groups. The distance is expressed by linguistic variables: very close, close, medium, far, too far. Because LIDAR has a visibility of $20 \mathrm{~m}$, a function between $0-20,000 \mathrm{~mm}$ is created. Except for the forward, backward, and stop functions, the robot can perform six different movements because of the Mecanum wheels. These movements are positioned to the negative and positive points as left and right. A rule base is developed in which the outputs are assigned according to the incoming data. The rule base consists of 45 rules (Table 2).

In the rule base, all possible inputs are evaluated, and output values related to an expert opinion are formed. In addition, if the width of the gap is smaller than the robot width and no space is available to go further, the robot stops. The fuzzy logic classifier

Table 2. Rule base

\begin{tabular}{|cccc}
\hline Gap & $\begin{array}{c}\text { Obstacle } \\
\text { angle }\end{array}$ & $\begin{array}{c}\text { Obstacle } \\
\text { distance }\end{array}$ & $\begin{array}{c}\text { Robot } \\
\text { movement }\end{array}$ \\
\hline Left & Left & Very close & Right side \\
\hline Left & Left & Close & Right cross \\
\hline Left & Left & Middle & Right cross \\
\hline Left & Left & Far & Right cross \\
\hline Left & Left & Too far & Straight \\
\hline Left & Middle & Very close & Left side \\
\hline Left & Middle & Close & Left cross \\
\hline Left & Middle & Middle & Left cross \\
\hline Left & Middle & Far & Left cross \\
\hline Left & Middle & Too far & Left cross \\
\hline Left & Right & Very close & Left side \\
\hline Left & Right & Close & Left cross \\
\hline Left & Right & Middle & Left cross \\
\hline Left & Right & Far & Left cross \\
\hline Left & Right & Too far & Left cross \\
\hline Middle & Left & Very close & Right side \\
\hline Middle & Left & Close & Right cross \\
\hline Middle & Left & Middle & Right cross \\
\hline Middle & Left & Far & Right cross \\
\hline Middle & Left & Too far & Straight \\
\hline Middle & Middle & Very close & Left side \\
\hline Middle & Middle & Close & Left cross \\
\hline Middle & Middle & Middle & Left cross \\
\hline & & & \\
\hline
\end{tabular}

\begin{tabular}{|cccc}
\hline Middle & Middle & Far & Left cross \\
\hline Middle & Middle & Too far & Left cross \\
\hline Middle & Right & Very close & Left side \\
\hline Middle & Right & Close & Left cross \\
\hline Middle & Right & Middle & Left cross \\
\hline Middle & Right & Far & Left cross \\
\hline Middle & Right & Too far & Straight \\
\hline Right & Left & Very close & Right side \\
\hline Right & Left & Close & Right cross \\
\hline Right & Left & Middle & Right cross \\
\hline Right & Left & Far & Right cross \\
\hline Right & Left & Too far & Right cross \\
\hline Right & Middle & Very close & Right side \\
\hline Right & Middle & Close & Right cross \\
\hline Right & Middle & Middle & Right cross \\
\hline Right & Middle & Far & Right cross \\
\hline Right & Middle & Too far & Right cross \\
\hline Right & Right & Very close & Left side \\
\hline Right & Right & Close & Left cross \\
\hline Right & Right & Middle & Left cross \\
\hline Right & Right & Far & Left cross \\
\hline Right & Right & Too far & Straight \\
\hline & & & \\
\hline
\end{tabular}

and control algorithms are coded in Python. The fuzzy logic classifier is created using the scikit-fuzzy library [18]. Because the output of the fuzzy logic used in the study determines the type of robot motion, the term fuzzy logic classifier is used instead of fuzzy logic controller. The employed fuzzy logic classifier actually controls the movement of the robot depending on the location between the robot and obstacle. Thus, we can say that it is used as a controller.

\section{Experimental Studies}

Experimental studies were carried out in the Mechatronics Engineering Laboratory of Karabuk University, Faculty of Technology Department. The experimental studies were performed under three different scenarios, which included scanning the area, avoiding static obstacles, and avoiding static/moving obstacles.

In this study, five experiments were performed under three different experimental setups. Fuzzy logic and a gap-tracking algorithm were used together in the experiments. Figure 8 shows the flowchart of the approach followed in the experiments. Figure 8 shows that the robot scanning functions of 


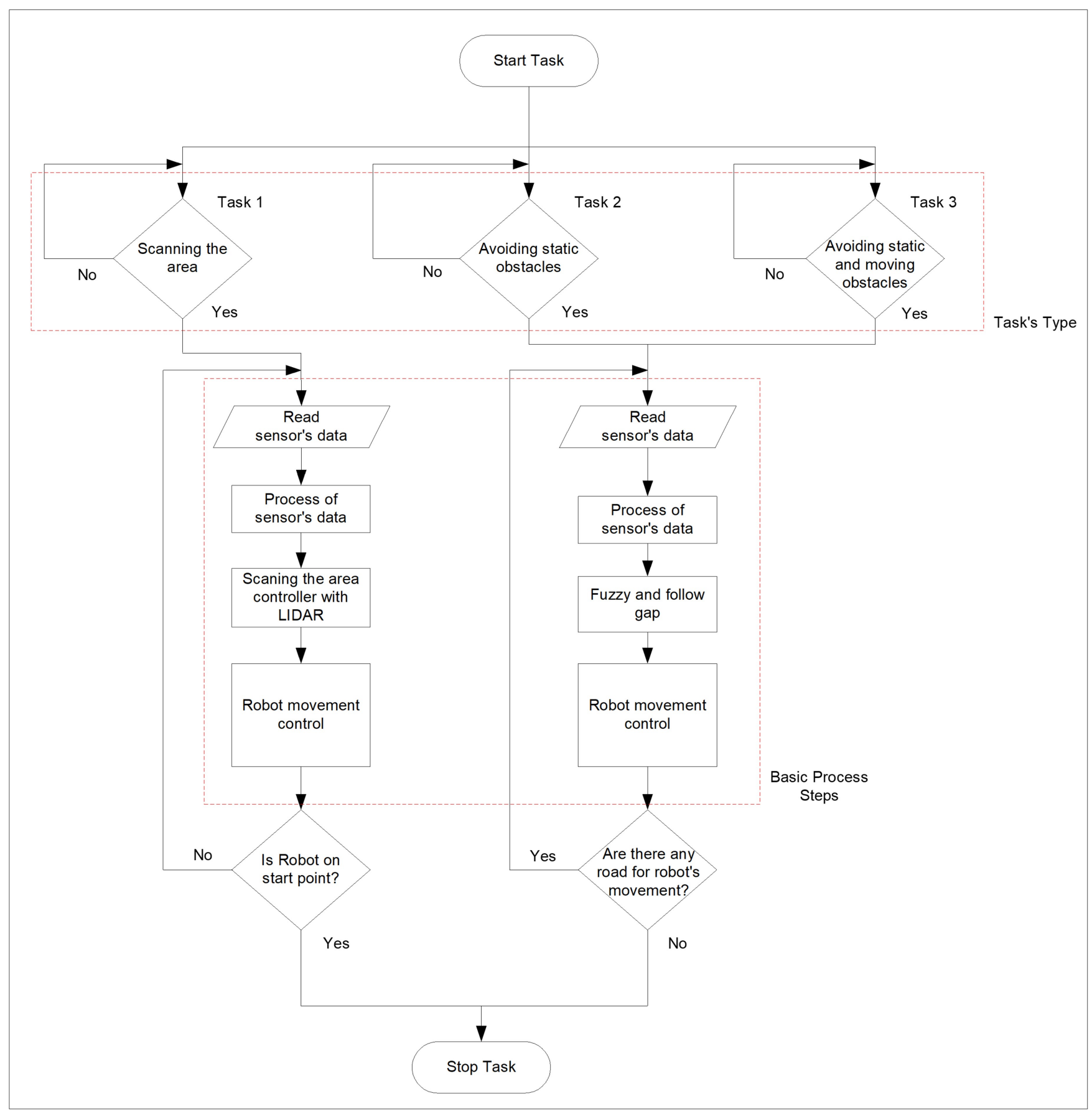

Figure 8. Flowchart of the approach followed in the experiments.

the environment were separate. However, to avoid fixed and movable obstacles, a common flowchart was used because the same flow was followed.

Figure 8 shows three different experiments such as scanning the robot environment and avoiding fixed and moving obstacles. Figure 8 shows the tracked methodology of the mobile robot to avoid obstacles based on the LIDAR information. In this methodology, different process steps were introduced depend- ing on the task types. Each experiment was repeated five times to increase the measurement accuracy.

Two obstacles were placed in front of the robot for detection. The environment measured by the robot could be given as a two-dimensional graph in terms of point-cloud distance angle (Figure 9). The two obstacles shown in Figure 8 were between $319^{\circ}-346^{\circ}$ and $12^{\circ}-39^{\circ}$, as shown in Figure 10 . The obstacles were located approximately $1 \mathrm{~m}$ from the robot. Each measure- 


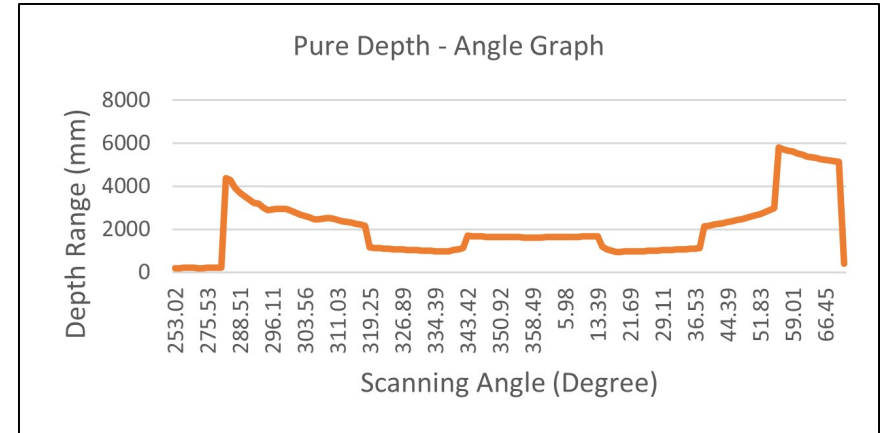

Figure 9. View of the LIDAR data.

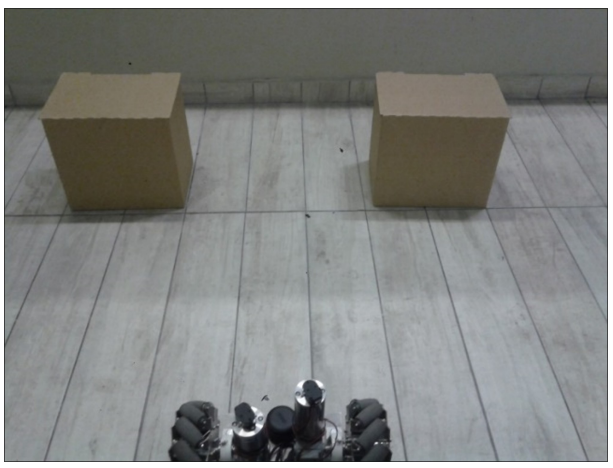

Figure 10. Environment where the data in Figure 9 are measured by LIDAR.

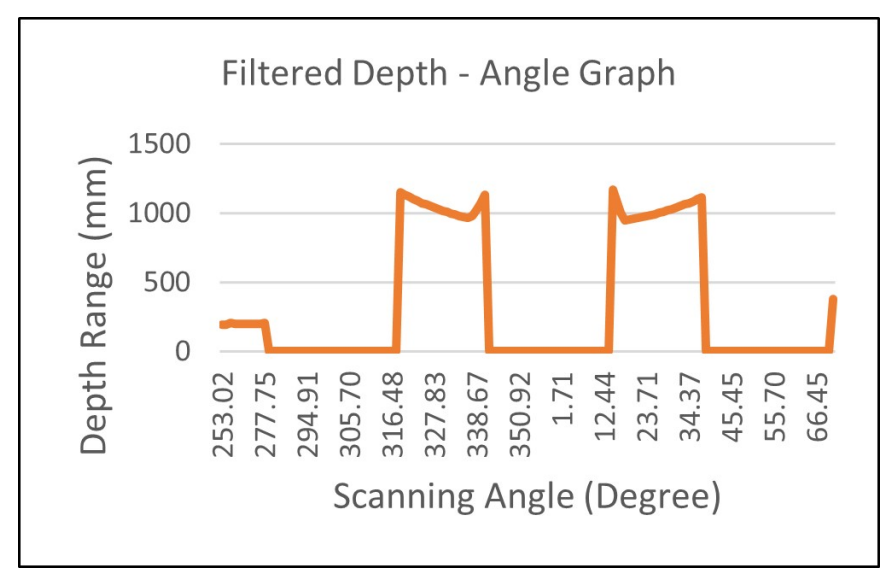

Figure 11. Filtered distance angle chart.

ment over a distance of more than $2 \mathrm{~m}$ is considered zero, and the graph shown in Figure 9 is similar to that shown in Figure 11.

Figure 10 shows that two obstacles were placed in front of the mobile robot. Figure 11 shows the results of these obstacles filtered after they were received by the LIDAR.

Figure 11 shows that obstacles appeared as a result of the applied filter.

\subsection{Field-Scanning Experiment of the Mecanum-Wheeled Mobile Robot}

In this experiment, our test found that the mobile-robot platform with Mecanum wheels could start to move in an area without hitting around and go back to where it started. We found that the robot could repeatedly perform the same movements. Images of the working environment are shown in Figure 12.

The robot was required to scan the working environment. The robot was expected to scan the working environment following the route shown in Figure 13. During the scanning process, the robot moved and maintained its position relative to the right wall. It also controlled the obstacles at its front and determined its movement. The experiments were repeated five times and recorded. Each repetition was done with a fully charged battery. The field-scanning experimental results are shown in Figure 13.

The floor was not completely smooth because the ambient floor was paved with cobblestones. In this case, the robot was exposed to vibrations from the Mecanum wheels as it passed through each cobblestone. This vibration created shifts in the robot movement. The robot position was corrected according to its distance from the wall. The robot moved left when an obstacle appeared in front. When it determined that the corridor was on its right side, it moved forward for 2 seconds, turned right, and moved forward to avoid the sharpness of the right turns. The robot tried to maintain a distance of 80 to $110 \mathrm{~cm}$ from the right wall.

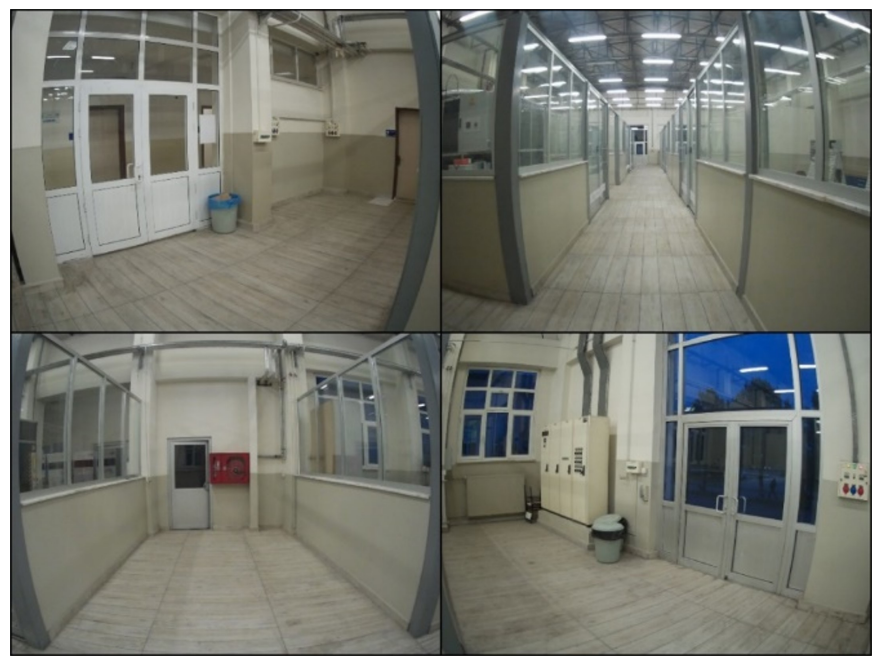

Figure 12. View of the laboratory environment where the field screening test is performed. 


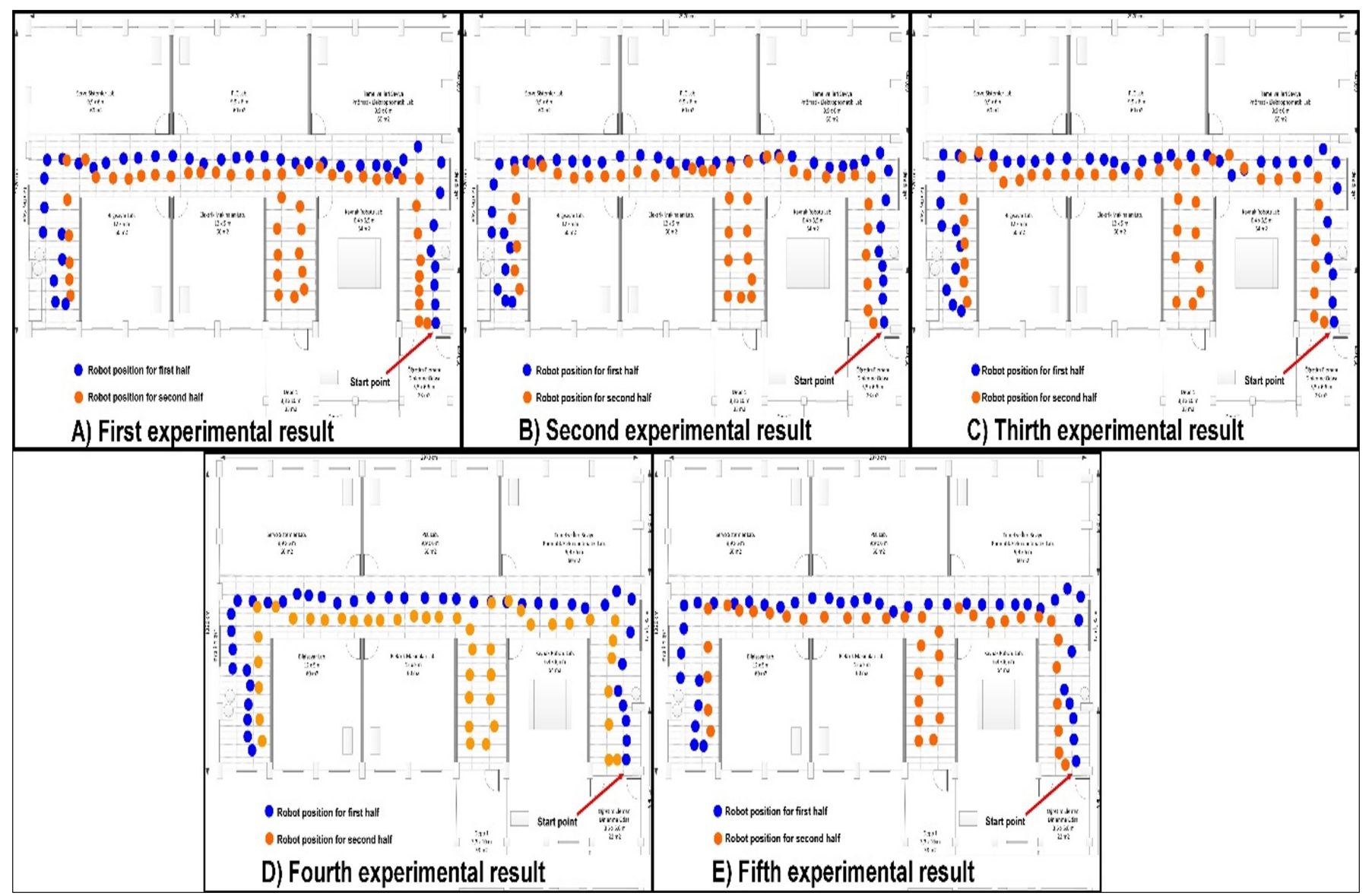

Figure 13. Results of the field-scan test.

\subsection{Mecanum-Wheeled Mobile-Robot Static-Obstacle- Avoidance Test}

In the experiment, the mobile robot consistently started from the same point to measure the robot response as observed. Carton boxes with the same dimensions were used as static obstacles. The dimensions of the carton boxes were $40 \times 40 \times 60 \mathrm{~cm}^{3}$. The static obstacles were positioned at different points to study the robot mobility. The obstacles were positioned as shown in Figure 14 (blue line) to test the robot ability to pass between two obstacles and avoid cross obstacles. The robot tried to pass the center of the gaps between the obstacles according to its algorithm. The expected movement of the robot is shown in Figure 14.

The experiments were repeated five times. The battery was fully charged. In all of the five repetitions, the robot was observed to avoid obstacles. The robot movements in all experiments are shown in Figure 14.

Figure 14 shows that in the experimental study, the robot avoided obstacles in all five repetitions. The advantage of the
Mecanum wheels was that the robot could perform cross and side movements to avoid cross obstacles.

\subsection{Static- and Mobile-Obstacle-Avoidance Test of the Mecanum-Wheeled Mobile Robot}

In this experiment, the ability of the mobile robot to avoid static and moving obstacles was investigated. In the experiment, the robot consistently started from the same point to investigate its response. Carton boxes from the static-barrier avoidance test were used as static obstacles. A training robot called mBot was used as a moving obstacle. The robot was covered with a cloth, as shown in Figure 15, to create an obstacle.

The moving obstacle moved to the right and left following the line shown in Figure 16. Figure 16 also shows the positions of the mobile and static obstacles in the static- and mobileobstacle-avoidance test environment.

The experiment was repeated five times, and it started with a full charge in the battery. The robot did not hit any static obstacles in the five experiments. In addition, it was able to 


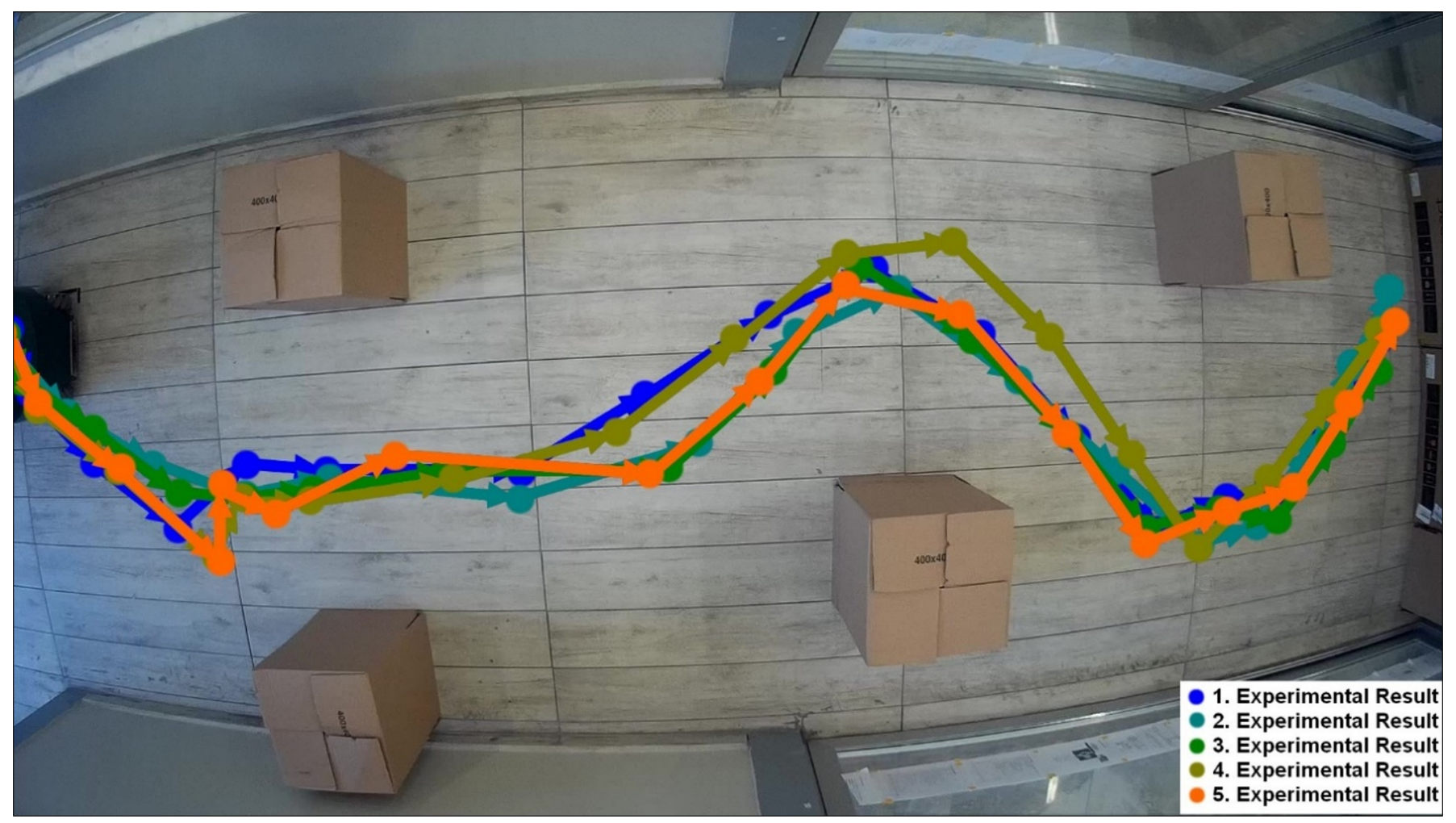

Figure 14. Results of the static-obstacle-avoidance tests.

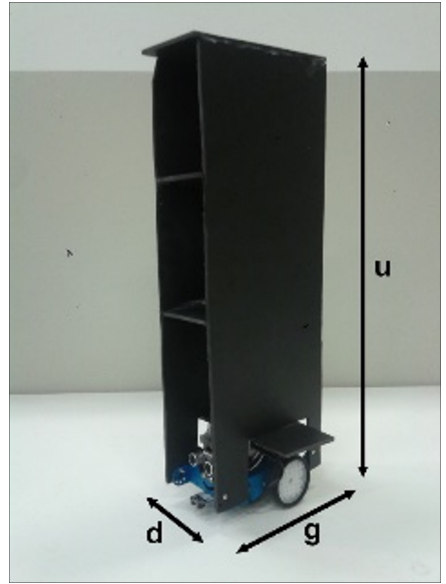

Figure 15. Moving obstacle.

complete the experiment by hitting the moving obstacle twice only. The first repetition of the static- and mobile-obstacleavoidance test is shown in Figure 17.

In the first repetition, the robot avoided both static and moving obstacles. Figure 11, which is reproduced in Figure 17, shows the moving obstacle at the right side of the robot. The robot moved toward the wide space on its left side. The robot successfully completed the movement toward the end of the

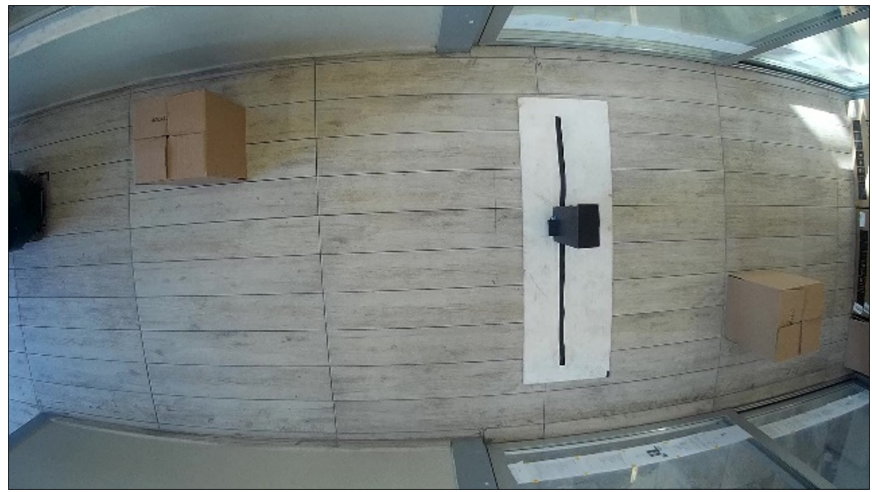

Figure 16. Static- and mobile-obstacle-avoidance test environment.

course while successfully avoiding the moving obstacle.

In the second repetition, the robot and moving obstacle collided. The robot managed to avoid the static obstacles. During the experiment, the moving obstacle remained at the left side of the robot. Therefore, the robot was directed toward the right side. However, the moving obstacle moved toward the robot. The robot, which moved to the right, collided with the moving obstacle. The robot completed the course by avoiding the static obstacle after passing the moving-obstacle zone.

In the third repetition, the robot and the moving obstacle 


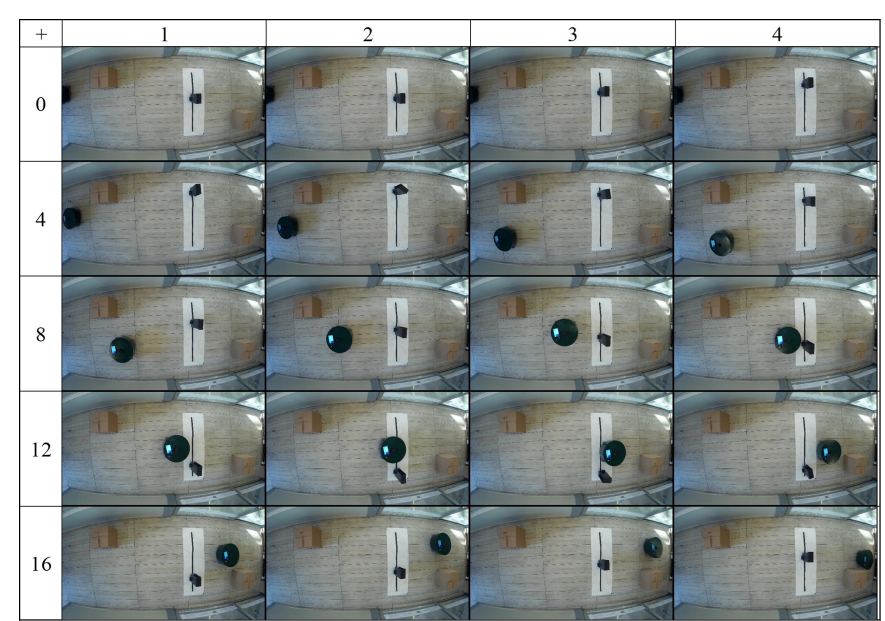

Figure 17. Static- and moving-obstacle-avoidance experiments results.

collided. The moving obstacle was at the right side of the robot. Then, the robot was directed to the left. However, the moving obstacle caught up with robot and moved to its front. The robot collided with the moving obstacle. The robot continued its movement after this collision, avoiding the static obstacles.

In the fourth repetition, no collision occurred. The robot reached the end of the track, avoiding the static and moving obstacles. When the robot encountered the moving obstacle, it detected a gap at its left side. It passed the left side of the moving obstacle and completed the course without any collision. Similarly, no collision occurred in the fifth repetition.

\section{Conclusion and Evaluation}

In this study, a mobile-robot platform with four Mecanum wheels was designed and manufactured. Mecanum wheels are known to provide versatility to robots. We observe that this mobility allows the robot to use multiple motion scenarios while avoiding obstacles. LIDAR technology is used to detect obstacles. Mobile robots usually know the map and its location in the map. In this study, the robot does not know its position and the map. Therefore, it avoids static and dynamic obstacles that may be encountered while moving freely in its environment. The robot was tested under three different scenarios. Each scenario was repeated five times. In the five repetitions, the robot consistently starts from the same position. In this manner, the accuracy is measured. In the first experiment, a successful result is obtained with $100 \%$ accuracy rate. The robot completes the field-scanning experiment at an average of 4 minutes 45 seconds. In the second experiment, a track, which consists of stationary obstacles, is completed by the robot with $100 \%$ accuracy. It completes the experiment at an average time of 38 seconds. In the third experiment, a track with both stationary and moving obstacles is completed by the robot. The third experiment is completed with $60 \%$ accuracy. The robot completes the experiment at an average time of 38 seconds.

To avoid obstacles, the gaps between the obstacles are measured during the movement, and the measured values and the distance and angle values of the nearest obstacle are evaluated using a fuzzy logic classifier. The follow-the-gap and fuzzy logic methods are used together in this study. The use of the follow-the-gap method and fuzzy logic classifier together is an original concept in this study. When the width of the largest gap is greater than that of the robot, the robot is directed to the gap. The robot, which is directed to the center of the gap, must also avoid obstacles during its movement. The gap angle, closest obstacle distance, and angle are used as inputs to the fuzzy logic classifier, and the output for the required robot movements is obtained.

In the Mecanum-wheeled mobile robots, the number of parameters to be controlled is large because the movement varies. Clearly, this problem cannot be solved using motor control only. In addition, because of the structure of the Mecanum wheels, slippage can occur during movement, which distorts the linearity of the path the robot follows to avoid obstacles. Therefore, in the Mecanum-wheeled mobile-robot work, the robot-position control makes obstacle avoidance more efficient. Owing to the structure of the Mecanum wheel, no large vibration can be experienced on smooth surfaces. However, too much vibration can occur on rough surfaces, which is undesirable. The use of Mecanum wheels with a suspension system can solve this problem.

LIDAR has a difficulty detecting glazed surfaces. LIDAR can sometimes see the glass, depending on the angle between the glass and the robot, and sometimes, it can see objects behind the glass. The presence of glazed sections in the laboratory causes deviations in the movement of the robot during the experiment To prevent deviations, the glazed surfaces within the LIDAR distance are covered with paper, which solves the problem of deviation in the robot movements.

Battery usage is very important in mobile robots because when the battery cannot supply sufficient power to the robot, problems such as reduction in the data read from the LIDAR and decrease in the robot speed can occur. To solve this problem, the battery-charge status must be continuously checked. The robot operation and task success are directly affected when the 
battery-charge level drops below a certain value.

\section{Conflict of Interest}

No potential conflict of interest relevant to this article was reported.

\section{Acknowledgements}

This work was supported as a KBU BAP-18-YL-157 coded BAP project. The authors would like to thank the Karabuk University Scientific Research Project Coordinator for its financial support.

\section{References}

[1] S. G. Tzafestas, Introduction to Mobile Robot Control. London, UK: Elsevier, 2014.

[2] M. O. Yatak, B. Goktas, and F. Duran, "Design and implementation of Android-based autonomous human tracking vehicle," Bilişim Teknolojileri Dergisi, vol. 11, no. 2, pp. 157-162, 2018. https://doi.org/10.17671/gazibtd.340566

[3] J. S. Keek, S. L. Loh, and S. H. Chong, "Comprehensive development and control of a path-trackable mecanumwheeled robot," IEEE Access, vol. 7, pp. 18368-18381, 2019. https://doi.org/10.1109/ACCESS.2019.2897013

[4] G. Li, Y. Tamura, A. Yamashita, and H. Asama, "Effective improved artificial potential field-based regression search method for autonomous mobile robot path planning," International Journal of Mechatronics and Automation, vol. 3, no. 3, pp. 141-170, 2013. https://doi.org/10.1504/ijma. 2013.055612

[5] J. H. Jung and D. H. Kim, "Local path planning of a mobile robot using a novel grid-based potential method," International Journal of Fuzzy Logic and Intelligent Systems, vol. 20, no. 1, pp. 26-34, 2020. https://doi.org/10. 5391/ijfis.2020.20.1.26

[6] J. Borenstein and Y. Koren, "The vector field histogramfast obstacle avoidance for mobile robots," IEEE Transactions on Robotics and Automation, vol. 7, no. 3, pp. 278-288, 1991. https://doi.org/10.1109/70.88137

[7] J. Oroko and B. Ikua, "Obstacle avoidance and path planning schemes for autonomous navigation of a mobile robot: a review," in Proceedings of the 2012 Mechanical Engineering Conference on Sustainable Research and Innovation, Juja, Kenya, 2012, pp. 314-318.

[8] J. Ng and T. Braunl, "Performance comparison of bug navigation algorithms," Journal of Intelligent and Robotic Systems, vol. 50, pp. 73-84, 2007. https://doi.org/10.1007/ s10846-007-9157-6

[9] V. Sezer and M. Gokasan, "A novel obstacle avoidance algorithm: "Follow the Gap Method"," Robotics and Autonomous Systems, vol. 60, no. 9, pp. 1123-1134, 2012. https://doi.org/10.1016/j.robot.2012.05.021

[10] A. Nasrinahar and J. H. Chuah, "Intelligent motion planning of a mobile robot with dynamic obstacle avoidance," Journal on Vehicle Routing Algorithms, vol. 1, pp. 89-104, 2018. https://doi.org/10.1007/s41604-018-0007-4

[11] D. Janglova, "Neural networks in mobile robot motion," International Journal of Advanced Robotic Systems, vol. 1, no. 1, pp. 15-22, 2004. https://doi.org/10.5772/5615

[12] S. L. Dickerson and B. D. Lapin, "Control of an omnidirectional robotic vehicle with Mecanum wheels," in Proceedings of the National Telesystems Conference (NTC), Atlanta, GA, 1991, pp. 323-328. https://doi.org/10.1109/ NTC.1991.148039

[13] A. Gfrerrer, "Geometry and kinematics of the Mecanum wheel," Computer Aided Geometric Design, vol. 25, no. 9, pp. 784-791, 2008. https://doi.org/10.1016/j.cagd.2008. 07.008

[14] L. C. Lin and H. Y. Shih, "Modeling and adaptive control of an omni-mecanum-wheeled robot," Intelligent Control and Automation, vol. 4, pp. 166-179, 2013. https://doi.org/ 10.4236/ica.2013.42021

[15] A. Ballagi and L. T. Koczy, "Fuzzy signature based mobil robot motion control system," in Proceedings of 2008 6th International Symposium on Applied Machine Intelligence and Informatics, Herlany, Slovakia, 2008, pp. 29-33. https: //doi.org/10.1109/SAMI.2008.4469193

[16] G. Csaba and Z. Vamossy, "Fuzzy based obstacle avoidance for mobil robots with Kinect sensor," in Proceedings of 2012 4th IEEE International Symposium on Logistics and Industrial Informatics, Smolenice, Slovakia, 2012, pp. 135-144. https://doi.org/10.1109/LINDI.2012.6319476 
[17] L. Gracia and J. Tornero, "Kinematic modeling of wheeled mobile robots with slip," Advanced Robotics, vol. 21, no. 11, pp. 1253-1279, 2007. https://doi.org/10.1163/ 156855307781503763

[18] Python Software Foundation, "scikit-fuzzy 0.4.2: fuzzy logic toolbox for Python,” Available https://pypi.org/ project/scikit-fuzzy/

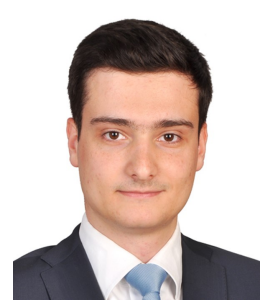

Musa Matli received his Master degree in Department of Mechatronics Engineering from the Karabuk University, Turkey. He is working on mecanum wheels mobile robots. His research interests include neural networks, fuzzy logic, mobil robots and control. His Ph.D. continue at Depertment of Mechatronics Engineering from the Karabuk University.

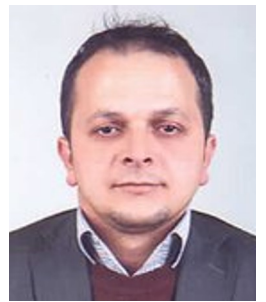

Ahmet Albayrak received his Ph.D. degree in Computer Engineering from the Karabük University, Turkey, in 2018. Currently he is working as Assistant Professor at the Düzce University, Turkey. Hismain research interests include machine learning, neural networks, deep learning and data science.

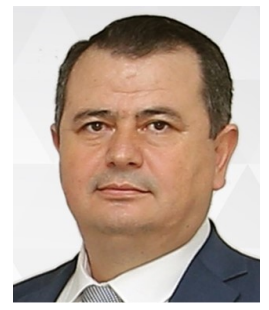

Raif Bayır received his Ph.D. degree in Department of Electronic and Computer Education Control Systems from the Gazi University, Turkey, in 2005. Currently he is working as Professor at the Karabük University, Turkey. His-main research interests include artificial intelligence, fault diagnosis and detection on electric machines, neural networks, robotics.

E-mail: rbayir@karabuk.edu.tr 